\title{
DISCOVERY OF THE GENUS WILKINSONELLUS MASON (HYMENOPTERA: BRACONIDAE) FROM INDIA
}

\author{
Zubair Ahmad 1, Kavita Pandey, Arshad Ali Haider and Shujauddin \\ Department of Zoology, Section of Entomology, Aligarh Muslim University, Aligarh, Uttar Pradesh 202002, India \\ E-mail: ${ }^{1}$ drazubair@yahoo.com
}

\begin{abstract}
A new species of the genus Wilkinsonellus, W. granulatus $s p$. nov., is described. This genus is reported for the first time from India, in Sholapur, Maharashtra.

KeYwords

Braconidae, first record, Hymenoptera, Microgastrinae, new species, Wilkinsonellus granulatus sp. nov.,

Abbreviations

OOL - Ocello-ocular line; POL - Post-ocellar line; AOL Anterior-ocellar line; ØOD - Diameter of an ocellus; ZDAMU -

Zoology Department, Aligarh Muslim University.
\end{abstract}

The genus Wilkinsonellus was described by Mason (1981) with Apanteles iphitus Nixon as its type species, comprising daira and henicorpus species group of Apanteles (Nixon, 1965). It is easily recognized by the shape of $\mathrm{T} 1$, sculpturing of propodeum; absence of an aerolete; short and solid hypopygium and short ovipositor sheaths. This is the first record of this genus from India and a new species is described.

\section{Wilkinsonellus granulatus sp. nov.}

(Figs. 1-3)

\section{Material examined}

Holotype: Female, 25.iii.1999, Solapur, Maharashtra, coll. Kapana Varshney, ZDAMU.

Paratype: 1 Female, with the same data as holotype.

\section{Diagnosis}

Female: Body length, $2.25 \mathrm{~mm}$; fore wing $2.2 \mathrm{~mm}$.

Head: Oval in anterior view, sub-rectangular in dorsal view with moderately long white pilosity including eyes; clypeus reticulate rugose, anterior tentorial pits large and about $0.75 \mathrm{x}$ as long as tentorio-ocular line; face reticulate rugose with a complete median dorsal node extending upto the clypeus; frons deeply concave behind antennal socket with some transverse striations; vertex and temple behind eyes somewhat indistinctly punctate with hairs; OOL 0.6x as long as POL; POL 1.5x as long as AOL; antennae 1.25x longer than body.

Mesosoma: Covered with sparse moderately long white pilosity except medial mesopleuron; mesoscutum reticulate with very fine granulate back ground sculpture, notauli faintly indicated by depressions posteriorly; disc of scutellum reticulate with very finely granulate back ground sculpture, with distinct up turned carinae on posterior margin; propodeum rugose with complete median carina, its spiracle ovoid in shape and enclosed by constulae; mesopleuron smooth and shiny medially, striate anteroventrally extending up to the mesosternum, epicnemial groove deep; sternaulus deep with 3-4 large fovea. Fore wing with even and dense pilosity; pterostigma about $3.1 \mathrm{x}$ as long as wide and about as long as $1-\mathrm{R} 1 ; \mathrm{r}$ about as long as width of
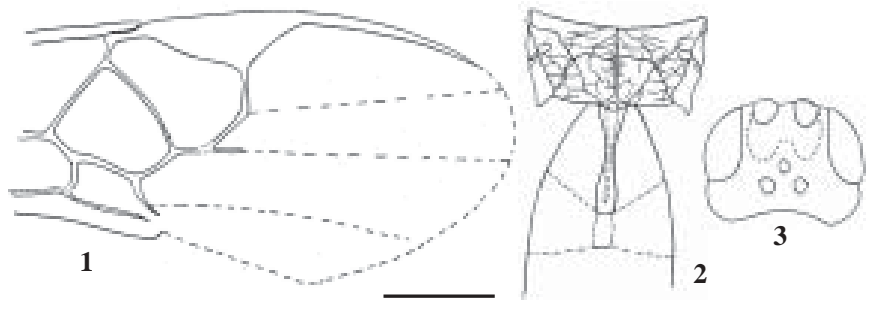

Figures. 1-3. Wilkinsonellus granulatus sp. nov. (female) 1 - Fore wing; 2 - Propodeum; 3 - Head, dorsal view. Scale $-0.5 \mathrm{~mm}$

pterostigma. Hind wing with vannal lobe convex and almost hairless. Hind coxae long and narrow in dorsal view, reaching well beyond posterior margin of $\mathrm{T} 3$, its surface reticulate with fine granulate background sculpture.

Metasoma: $\mathrm{T} 1$ about $4 \mathrm{x}$ as long as maximum width with a medial longitudinal furrow; T2 with indistinct narrow elongate median field; hypopygium smooth and hairless; ovipositor sheaths short with even width in lateral view.

Colour: Dull reddish-brown except for the following: scape, fore leg, mid leg, palpi, T1, T2, laterotergite 1, laterotergite 2, hypopygium are yellowish; hind-femur, tibia, tarsi, T3-T6, ovipositor sheaths and apical segments of antennae brownish; face, clypeus, checks are yellowish-brown; wings hyaline; venation and pterostigma brown.

Male: Unknown.

Host: Unknown.

\section{Remarks}

Wilkinsonellus granulatus, sp. nov. closely resembles W. thyone (Nixon) but differs in having mesoscutum, scutellum and hind coxae reticulate with finely granulate background sculpture (mesoscutum, scutellum dull in thyone) and frons strongly depressed behind antennal socket with few transverse striations (frons smooth in thyone).

\section{REFERENCES}

Mason, W.R.M. (1981). The polyphyletic nature of Apanteles Foerster (Hymenoptera: Braconidae) a phylogeny and reclassification of Microgasterinae. Memoires of the Entomological Society of Canada 115: 1-147.

Nixon, G.E.J. (1965). A reclassification of the tribe Microgastrinae (Hymenoptera: Braconidae). Bulletin of British Museum (Natural History) Entomology (Supplement) 2: 1-284.

\section{ACKnowledgement}

The authors are thankful to Professor M.Hayat for reviewing the manuscript and offering useful suggestions. 